\title{
Ciberbullying en adolescentes y jóvenes del País Vasco: Cambios con la edad
}

\author{
Maite Garaigordobil
}

Facultad de Psicologia. Universidad del País V asco (España).

\begin{abstract}
Resumen: El estudio tuvo como objetivo analizar cambios con la edad en ciberbullying. La muestra fue de 3026 participantes del País Vasco de 12 a 18 años. Se administró el Test Cyberbullying para evaluar la frecuencia con la que 15 conductas han sido sufridas, realizadas y observadas durante el último año, y permite obtener 4 indicadores psicométricos: victimización, agresión, observación, victimización-agresiva. Los resultados evidenciaron 1) Similar porcentaje de víctimas de 12 a 18 años en las 15 conductas; 2) Un aumento de agresores a medida que aumenta la edad en 5 conductas (difundir fotos comprometidas, chantajear-amenazar, acosar sexualmente, robar la contraseña, amenazar de muerte); 3) Un incremento de observadores con la edad en 12 conductas (enviar mensajes ofensivos, llamadas ofensivas, difundir fotos comprometidas, hacer fotos robadas para humillar, llamadas anónimas para asustar, suplantar la personalidad, robo de contraseña, trucar fotos para humillar, aislar en redes sociales, chantajear para obligar a hacer algo, amenazar de muerte, difamar/difundir rumores); y 4) Un aumento con la edad en la media de conductas de ciberbullying que los agresores realizan, y que los testigos ven. El estudio provee información sobre la significativa prevalencia del ciberbullying durante la adolescencia y juventud. La discusión se centra en la necesidad de implementar programas de intervención psicoeducativa para prevenir la violencia entre iguales. Palabras clave: Ciberbullying; violencia; edad; adolescencia; juventud.
\end{abstract}

\section{Introducción}

En los últimos años, el interés y la preocupación social por las conductas violentas entre iguales, tanto, "cara a cara" (bullying), como a través de las TIC -Tecnologías de la Información y de la Comunicación- (ciberbullying) ha ido incrementándose, y este interés y preocupación están en la base del estudio. El ciberbullying consiste en utilizar las TIC, principalmente Internet y el móvil, para ejercer acoso entre iguales. Según Smith et al. (2008), el ciberbullying es una conducta agresiva e intencional que se repite de forma frecuente en el tiempo mediante el uso, por un individuo o grupo, de dispositivos electrónicos sobre una víctima que no puede defenderse por sí misma fácilmente. Las vías utilizadas son variadas: mensajes de texto (SMS-de móvil), acoso telefónico (llamadas anónimas al móvil...), grabaciones de agresiones físicas o vejaciones que son difundidas vía móvil o Internet, acoso mediante fotografías y vídeos que se difunden vía móviles o se suben a You Tube, correos electrónicos, en las redes sociales, páginas web... El rápido desarrollo y crecimiento de esta nueva forma de acoso ha generado la urgente necesidad de su estudio (Garaigordobil, 2011).

La violencia entre iguales tiene consecuencias perniciosas para todos los implicados pero con distintos síntomas y niveles de sufrimiento. Aunque los efectos más acusados se muestran en las víctimas, los agresores y los observadores

* Dirección para correspondencia [Correspondence address]:

Maite Garaigordobil. Avda. de Tolosa 70. 20018 San Sebastián (España).

E-mail: maite.garaigordobil@ehu.es
Title: Cyberbullying in adolescents and youth in the Basque Country: Changes with age.

Abstract: The study aimed to analyze changes with age in cyberbullying. The sample comprised 3,026 participants from the Basque Country, aged from 12 to 18 years. The Cyberbullying Test was administered to assess the frequency with which 15 behaviors had been received, performed and observed during the past year, and to identify 4 psychometric indicators: victimization, perpetration, observation, and aggressive victimization. The results showed: 1) Similar percentages of victims from 12 to 18 years in the 15 conducts; 2) An increase of aggressors with age in 5 conducts (broadcasting private photos, blackmailing-threatening, sexual harassment, stealing a password, death threats); 3) An increase of observers with age in 12 conducts (sending offensive messages, offensive calls, broadcasting private photos/videos, taking photos in dressing rooms/beach... to humiliate; anonymous frightening calls, identity theft, stealing a password, rigging photos to humiliate, isolating on social networks, blackmail to obligate to do something, death threats, slandering/spreading rumors); and 4) An increase with age of the average of aggressive conducts performed by the aggressors and seen by the observers. The study provides information on the significant prevalence of cyberbullying during adolescence and youth. The discussion focuses on the need to implement psychoeducational intervention programs to prevent peer violence.

Key words: Cyberbullying; violence; age; adolescence; youth.

también son receptores de aprendizajes y hábitos negativos. Todos los implicados en situaciones de maltrato, en cualquiera de los roles, están en mayor situación de riesgo de sufrir desajustes psicosociales y trastornos psicopatológicos en la adolescencia y en la vida adulta (Garaigordobil, 2011).

Las negativas consecuencias que tiene el cyberbullying para las víctimas (ansiedad, depresión, estrés, miedo, ira, frustración, somatizaciones, trastornos del sueño, disminución del rendimiento escolar...), y los agresores (falta de empatía, conducta antisocial-delictiva, problemas con alcohol/drogas, con el rendimiento académico...), así como la prevalencia evidenciada en los distintos estudios, permite enfatizar la importancia de identificar el porcentaje de niños, adolescentes y jóvenes involucrado en este tipo de acoso (Garaigordobil y Oñederra, 2010).

El interés de la presente investigación se centra en analizar cambios con la edad en conductas de violencia entre iguales a través de las TIC, es decir, el ciberbullying (cuando se detecta que estas conductas se producen bastantes veces o siempre durante el último año), teniendo en cuenta la información aportada desde diferentes roles implicados: víctimas, agresores y observadores. Este análisis del cambio con la edad será útil para clarificar si las conductas violentas a través de las TIC, las conductas de ciberbullying, se mantienen estables en el tiempo, lo que enfatizaría la necesidad de prevención/intervención durante toda la escolarización, o por el contrario estas conductas disminuyen evolutivamente y, por consiguiente, no debe sobredimensionarse la problemática.Para analizar la evolución del ciberbullying durante la adolescencia y la juventud, se realizó una revisión de los estudios 
llevados a cabo durante los últimos años. En victimización, la mayoría de los estudios no encuentra diferencias en función de la edad durante la adolescencia (Bauman, 2010; Gofin y Avitzour, 2012; Juvoven y Gross, 2008; Katzer, Fetchenhauer y Belschak, 2009; Lauren y Ratliffe, 2011; Ortega, Calmaestra y Mora-Merchán, 2008; Slonje y Smith, 2008; Walrave y Heirman, 2011) y juventud (Hemphill, Tollit y Kotevski, 2012). Sin embargo, hay estudios con adolescentes (12-16 años) que han hallado menor porcentaje de víctimas con la edad (Dehue, Bolman y Völlink, 2008; Mishna, Khoury-Kassabri, Gadalla y Daciuk, 2012), menor nivel de victimización (Moore, Huebner y Hills, 2012), mientras que otros estudios con muestras de 7 a 18 años han evidenciado un aumento de las víctimas con la edad (Hinduja y Patchin, 2008; Kowalski y Limber, 2007; Monks, Robinson y Worlidge, 2012). Además, hay trabajos que muestran una relación curvilínea entre victimización y edad, observando menos víctimas a los 10-11 años, un pico a los 13-14 y un descenso a los 16-17 años (Cassidy, Jackson y Brown, 2009; Sakellariou, Carroll y Houghton, 2012).

En perpetración, muchos estudios realizados con participantes de 11 a 18 años concluyen que a medida que aumenta la edad aumenta el número de agresores (Hinduja y Patchin, 2008; Kowalski y Limber, 2007; Mishna et al., 2012; Raskauskas y Stoltz, 2007; Smith et al., 2008; Walrave y Heirman, 2011), y el nivel de agresión (Bauman, 2010; Lauren y Ratliffe, 2011; Ybarra y Mitchell, 2007). Aún así, algunos autores no han hallado diferencias en función de la edad, ya sea con niños o adolescentes (Monks et al., 2012; Ortega et al., 2008; Slonje y Smith, 2008), o han hallado una disminución de los agresores de educación primaria a secundaria (Dehue et al., 2008). Otros estudios han mostrado menor porcentaje de agresores a los 10-11 años, una elevación a los 12-14 y un descenso a los 16-17 años (Calvete, Orue, Estévez, Villardón y Padilla 2010; Williams y Guerra, 2007). Los escasos estudios que recogen información de los observadores (ÁlvarezGarcía et al., 2011) han hallado que el nivel de violencia a través de las TIC percibido por el alumnado aumentó hasta los 13-14 años, disminuyendo posteriormente.

Teniendo en cuenta los diferentes resultados obtenidos en las investigaciones que han analizado los cambios en el ciberbullying durante la adolescencia y la juventud, este estudio tiene tres objetivos: 1) identificar el porcentaje de víctimas, agresores, observadores en quince conductas de ciberbullying, en distintos rangos de edad (12-13, 14-15, 16-18); 2) calcular los niveles de victimización, perpetración, observación y victimización-agresiva en cada rango de edad, es decir, la media de las conductas sufridas, realizadas y/o observadas de 12 a 18 años; y 3) identificar las conductas que aumentan con la edad desde la opinión de las víctimas, agresores y observadores.

El estudio tiene como finalidad arrojar luz en torno a la evolución del ciberbullying durante la adolescencia y juventud, formulando 3 hipótesis. H1: Pese a la maduración personal y social que se produce de 12 a 18 años, tanto el porcentaje de víctimas de ciberbullying en las diferentes conduc- tas evaluadas, como la media de conductas de acoso que sufren las víctimas (nivel de victimización) será similar entre los 12 y los 18 años, ni aumentará ni disminuirá, se mantendrá estable. H2: El porcentaje de perpetradores en los tres rangos de edad, así como la media de conducta agresiva ejercida por los mismos hacia las víctimas (nivel de agresión) será similar de 12 a 18 años. H3: El porcentaje de participantes que observan conductas de ciberbullying de 12 a 18 años será similar, y la media de conductas de ciberbullying observadas por los observadores/testigos se mantendrá estable durante estas edades.

\section{Método}

\section{Participantes}

La muestra estaba constituida por 3026 participantes del País Vasco de 12 a 18 años (48.5\% varones, 51.5\% mujeres). Se distribuían en 3 grupos de edad: 12-13 (35.1\%), 14-15 $(36.2 \%)$ y $16-18(28.8 \%)$. La frecuencia y porcentaje de varones y mujeres en cada rango de edad puede observarse en la Tabla 1. Los participantes, inscritos en 10 centros del País Vasco, públicos $(45.6 \%)$ y privados $(54.4 \%)$, cursaban Educación Secundaria Obligatoria (75.4\%) y Bachiller (24.6\%). En concreto, ESO 1: 574 (25.1\%), ESO 2: 606 (26.5\%), ESO 3: 540 (23.7\%), ESO 4: 563 (24.7\%), y Bachiller (24.6\%). Para seleccionar una muestra representativa de los estudiantes del País Vasco, se utilizó una técnica de muestreo estratificado, proporcional y aleatorio, teniendo en cuenta la proporcionalidad de centros en cada provincia y el equilibrio de distintas condiciones (nivel socio-económico-cultural, tipo de red...).

Tabla 1. Frecuencia y porcentaje de varones y mujeres en los tres grupos de edad.

\begin{tabular}{lcccc}
\hline & $12-13$ años & $14-15$ años & $16-18$ años & Total \\
\hline Varón & $543(51.2 \%)$ & $536(49 \%)$ & $390(44.8 \%)$ & $1469(48.5 \%)$ \\
Mujer & $518(48.8 \%)$ & $558(51 \%)$ & $481(55.2 \%)$ & $1557(51.5 \%)$ \\
Total & $1061(100 \%)$ & $1094(100 \%)$ & $871(100 \%)$ & $3026(100 \%)$ \\
\hline
\end{tabular}

\section{Materiales}

Test Cyberbullying (Garaigordobil, 2013). Instrumento estandarizado con garantías de fiabilidad y validez que evalúa 15 conductas de ciberbullying (ver Anexo 1). Contiene 45 ítems, agrupados en torno al rol que se desempeña en la situación de acoso: víctima, perpetrador y observador. Los participantes informan de la frecuencia con la que han sufrido, ejercido o visto en el último año las 15 conductas de ciberbullying. En la corrección se puntúa cada conducta (nun$\mathrm{ca}=0$, algunas veces $=1$, bastantes veces $=2$, siempre $=3$ ), y se obtiene una puntuación directa total en cada rol. El test aporta puntuaciones percentiles en 4 índices: nivel de victimización, agresión, observación y victimización-agresiva. Los datos obtenidos con la muestra de tipificación de la prueba confirmaron elevada consistencia interna global del 
instrumento $(\alpha=.91)$, así como en sus 3 factores: nivel de cybervictimización $(\alpha=.82)$, nivel de cyberagresión $(\alpha=.91)$ y nivel de cyberobservación $(\alpha=.87)$. El análisis factorial confirmó una estructura configurada por 3 factores que explican el $40.15 \%$ de la varianza. Estudios de validez convergente mostraron correlaciones positivas entre agresión y resolución agresiva de conflictos, neuroticismo, conducta antisocial, problemas escolares, trastornos psicopatológicos... y correlaciones negativas con empatía, responsabilidad, regulación emocional, y adaptación social.

\section{Procedimiento}

Utilizando un diseño descriptivo y correlacional de corte transversal, en primer lugar, se envió una carta a los centros educativos seleccionados explicando la investigación. Posteriormente, se contactó telefónicamente con la dirección de los centros, y con aquellos que aceptaron participar se concertó una entrevista en la que se explicó el proyecto con más detalle, y se entregaron los consentimientos informados para padres y participantes. Después de recibir los consentimientos firmados, un equipo compuesto por la investigadora principal del estudio y 10 Licenciados-as en Psicología y estudiantes del programa de doctorado de la Facultad de Psicología formados con esta finalidad, se desplazaron a los centros y administraron el Test Cyberbullying. La evaluación se llevó a cabo en una sesión de evaluación de 30 minutos de duración. El estudio cumplió los valores éticos requeridos en la investigación con seres humanos, habiendo sido evaluado favorablemente por la Comisión Universitaria de Ética de la Investigación y la Docencia de la Universidad del País Vasco
(CEISH/112/2012). El protocolo del consentimiento informó sobre los objetivos del estudio, el carácter de voluntariedad, la protección de datos personales y garantías de confidencialidad, el derecho a la información, y la posibilidad de abandonar el estudio en cualquiera de sus fases.

\section{Análisis de datos}

Para analizar diferencias en función de la edad se realizaron análisis de contingencia de las respuestas (nunca/algunas veces/bastantes veces/siempre) en los 3 grupos de edad, y en cada rol (víctima, agresor, observador), obteniendo la chi cuadrado de Pearson para cada conducta. Posteriormente, después de llevar a cabo los supuestos básicos (normalidad, homocedasticidad...), con la finalidad de averiguar si la media de conductas de ciberbullying varía con la edad, se realizaron análisis de varianza multivariantes y univariantes con las puntuaciones de los 4 indicadores: victimización, agresión, observación, victimización-agresiva. Los análisis estadísticos fueron realizados con el programa SPSS 20.0.

\section{Resultados}

\section{Porcentaje de víctimas, agresores y observadores de 12 a 18 años}

Los resultados de los análisis de contingencia (frecuencias, porcentajes, chi cuadrado de Pearson) en las 15 conductas para cada rol, en los tres grupos de edad, se presentan en las Tablas 2,3 y 4.

Tabla 2. Víctimas de Ciberbullying: Frecuencia y porcentaje de participantes que han sufrido ciberbullying en el último año en función de la edad.

\begin{tabular}{|c|c|c|c|c|c|c|c|c|c|c|}
\hline \multirow[t]{3}{*}{ Conductas de Ciberbullying } & \multicolumn{3}{|c|}{ Algunas veces } & \multicolumn{3}{|c|}{ Bastantes veces } & \multicolumn{3}{|c|}{ Siempre } & \multirow[t]{3}{*}{$\chi^{2}$} \\
\hline & $12-13$ & $14-15$ & $16-18$ & $12-13$ & $14-15$ & $16-18$ & $12-13$ & $14-15$ & $16-18$ & \\
\hline & $\mathrm{F}(\%)$ & $\mathrm{F}(\%)$ & $\mathrm{F}(\%)$ & $\mathrm{F}(\%)$ & $\mathrm{F}(\%)$ & $\mathrm{F}(\%)$ & $\mathrm{F}(\%)$ & $\mathrm{F}(\%)$ & $\mathrm{F}(\%)$ & \\
\hline 1. Mensajes ofensivos/insultantes móvil/internet & $90(8.5)$ & $84(7.7)$ & $71(8.2)$ & $8(0.8)$ & $7(0.6)$ & $1(0.1)$ & $2(0.2)$ & $2(0.2)$ & $1(0.1)$ & $4.80 \mathrm{~ns}$ \\
\hline 2. Llamadas ofensivas/insultantes móvil/internet & $39(3.7)$ & $54(4.9)$ & $50(5.7)$ & $9(0.8)$ & $6(0.5)$ & $2(0.2)$ & $1(0.1)$ & $1(0.1)$ & $1(0.1)$ & $7.88 \mathrm{~ns}$ \\
\hline 3. Agredir, grabar y colgar en Internet & $8(0.8)$ & $13(1.2)$ & $12(1.4)$ & $0(0.0)$ & $1(0.1)$ & $1(0.1)$ & $0(0.0)$ & $2(0.2)$ & $1(0.1)$ & $4.86 \mathrm{~ns}$ \\
\hline 4. Difundir fotos/vídeos privados & $30(2.8)$ & $44(4.0)$ & $45(5.2)$ & $5(0.5)$ & $1(0.1)$ & $1(0.1)$ & $3(0.3)$ & $1(0.1)$ & $1(0.1)$ & $2.32 \mathrm{~ns}$ \\
\hline 5. Fotografiar en vestuarios, playa... y difundir & $19(1.8)$ & $17(1.6)$ & $12(1.4)$ & $3(0.3)$ & $3(0.3)$ & $1(0.1)$ & $3(0.3)$ & $2(0.2)$ & $0(0.0)$ & $3.62 \mathrm{~ns}$ \\
\hline 6. Llamadas anónimas para asustar & $84(7.9)$ & $95(8.7)$ & $88(10.1)$ & $7(0.7)$ & $8(0.7)$ & $9(1.0)$ & $4(0.4)$ & $1(0.1)$ & $1(0.1)$ & $6.51 \mathrm{~ns}$ \\
\hline 7. Chantajear/amenazar mediante llamadas/mensajes & $42(4.0)$ & $34(3.1)$ & $30(3.4)$ & $4(0.4)$ & $4(0.4)$ & $5(0.6)$ & $2(0.2)$ & $0(0.0)$ & $0(0.0)$ & $5.49 \mathrm{~ns}$ \\
\hline 8. Acosar sexualmente móvil/internet & $26(2.5)$ & $29(2.7)$ & $33(3.8)$ & $1(0.1)$ & $3(0.3)$ & $4(0.5)$ & $2(0.2)$ & $3(0.3)$ & $1(0.1)$ & $2 \mathrm{~ns}$ \\
\hline 9. Suplantar la personalidad & $78(7.4)$ & $68(6.2)$ & $52(6.0)$ & $8(0.8)$ & $6(0.5)$ & $1(0.1)$ & $2(0.2)$ & $1(0.1)$ & $2(0.2)$ & $6.56 \mathrm{~ns}$ \\
\hline 10. Robar la contraseña & $81(7.6)$ & $102(9.3)$ & $92(10.6)$ & $10(0.9)$ & $4(0.4)$ & $9(1.0)$ & $2(0.2)$ & $1(0.1)$ & $2(0.2)$ & $9.31 \mathrm{~ns}$ \\
\hline 11. Trucar fotos/vídeos y difundir & $16(1.5)$ & $19(1.7)$ & $10(1.1)$ & $3(0.3)$ & $1(0.1)$ & $0(0.0)$ & $1(0.1)$ & $1(0.1)$ & $0(0.0)$ & $5.08 \mathrm{~ns}$ \\
\hline 12. Aislar en redes sociales & $33(3.1)$ & $19(1.7)$ & $20(2.3)$ & $2(0.2)$ & $4(0.4)$ & $5(0.6)$ & $0(0.0)$ & $0(0.0)$ & $1(0.1)$ & $8.82 \mathrm{~ns}$ \\
\hline 13. Chantajear para hacer algo y no difundir intimidad & $24(2.3)$ & $24(2.2)$ & $17(2.0)$ & $2(0.2)$ & $1(0.1)$ & $2(0.2)$ & $1(0.1)$ & $2(0.2)$ & $0(0.0)$ & $2.48 \mathrm{~ns}$ \\
\hline 14. Amenazar de muerte & $17(1.6)$ & $13(1.2)$ & $20(2.3)$ & $4(0.4)$ & $1(0.1)$ & $3(0.3)$ & $0(0.0)$ & $2(0.2)$ & $1(0.1)$ & $7.53 \mathrm{~ns}$ \\
\hline 15. Difamar y crear rumores para desprestigiar & $76(7.2)$ & $84(7.7)$ & $72(8.3)$ & $6(0.6)$ & $15(1.4)$ & $5(0.6)$ & $4(0.4)$ & $2(0.2)$ & $3(0.3)$ & $6.85 \mathrm{~ns}$ \\
\hline
\end{tabular}

Notas: ns $=$ no significativo. $\chi^{2}=$ Chi cuadrado. Los resultados en "Nunca" son la diferencia de los expuestos en la tabla. La $\chi^{2}$ se calcula con las 4 formas de respuestas. 
Tabla 3. Agresores de Ciberbullying: Frecuencia y porcentaje de participantes que han perpetrado ciberbullying en el último año en función de la edad. Conductas de Ciberbullying $\quad$ Algunas veces $\quad$ Bastantes veces $\chi^{2}$

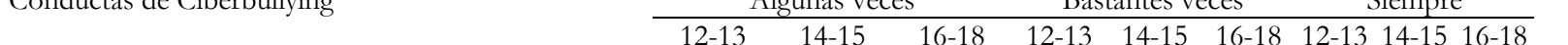

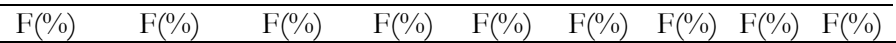

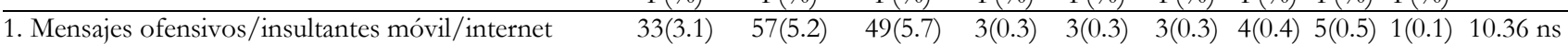

2. Llamadas ofensivas/insultantes móvil/internet

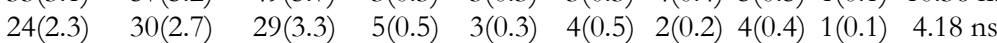

3. Agredir, grabar y colgar en Internet $\begin{array}{llllllllll}8(0.8) & 6(0.5) & 7(0.8) & 5(0.5) & 1(0.1) & 3(0.3) & 0(0.0) & 5(0.5) & 0(0.0) & 12.06 \mathrm{~ns}\end{array}$

4. Difundir fotos/vídeos privados

5. Fotografiar en vestuarios, playa... y difundir $\begin{array}{lllllllllll}12(1.1) & 21(1.9) & 22(2.5) & 2(0.2) & 2(0.2) & 1(0.1) & 0(0.0) & 4(0.4) & 0(0.0) & 12.58^{*}\end{array}$ $\begin{array}{llllllllll}12(1.1) & 13(1.2) & 12(1.4) & 2(0.2) & 0(0.0) & 2(0.2) & 0(0.0) & 4(0.4) & 0(0.0) & 9.65 \mathrm{~ns}\end{array}$

6. Llamadas anónimas para asustar

$\begin{array}{llllllllll}46(4.3) & 63(5.8) & 60(6.9) & 4(0.4) & 7(0.6) & 7(0.8) & 1(0.1) & 4(0.4) & 2(0.2) & 9.43 \\ \mathrm{~ns}\end{array}$

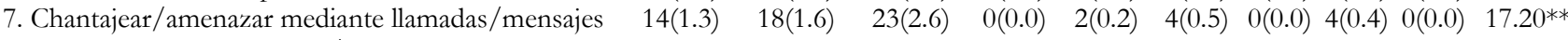

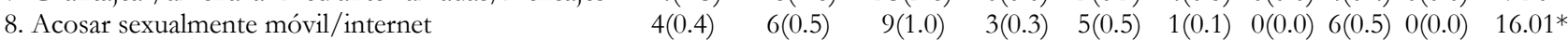

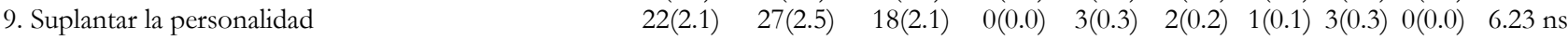

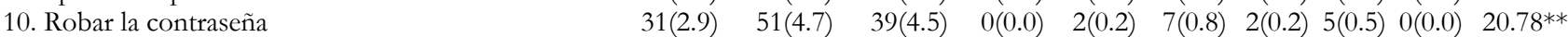

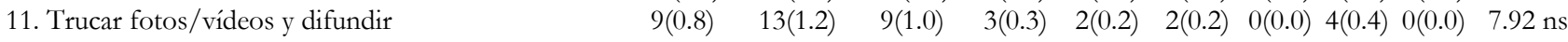

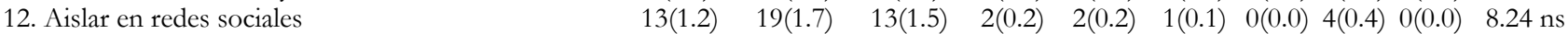

$\begin{array}{llllllllll}\text { 13. Chantajear para hacer algo y no difundir intimidad } \quad 6(0.6) & 7(0.6) & 8(0.9) & 0(0.0) & 6(0.5) & 2(0.2) & 0(0.0) & 3(0.3) & 1(0.1) & 10.25 \mathrm{~ns}\end{array}$

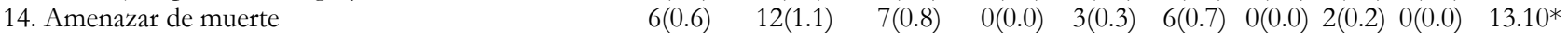

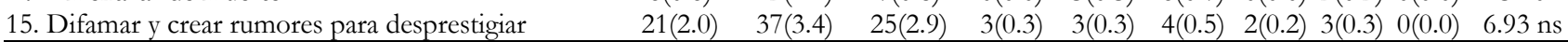

Notas: $\chi^{2}=$ Chi cuadrado. ${ }^{*} p<.05,{ }^{* *} p<.01, \mathrm{~ns}=$ no significativo. Los resultados en "Nunca" son la diferencia de los expuestos en la tabla. La $\chi^{2}$ se calcula con las 4 formas de respuestas.

Tabla 4. Observadores de Ciberbullying: Frecuencia y porcentaje de participantes que han observado ciberbullying en el último año en función de la edad.

\begin{tabular}{|c|c|c|c|c|c|c|c|c|c|c|}
\hline \multirow[t]{3}{*}{ Conductas de Ciberbullying } & \multicolumn{3}{|c|}{ Algunas veces } & \multicolumn{3}{|c|}{ Bastantes veces } & \multicolumn{3}{|c|}{ Siempre } & \multirow[t]{3}{*}{$\chi^{2}$} \\
\hline & $12-13$ & $14-15$ & $16-18$ & $12-13$ & $14-15$ & $16-18$ & $12-13$ & $14-15$ & $16-18$ & \\
\hline & $\mathrm{F}(\%)$ & $\mathrm{F}(\%)$ & $\mathrm{F}(\%)$ & $\mathrm{F}(\%)$ & $\mathrm{F}(\%)$ & $\mathrm{F}(\%)$ & $\mathrm{F}(\%)$ & $\mathrm{F}(\%)$ & $\mathrm{F}(\%)$ & \\
\hline 1. Mensajes ofensivos/insultantes móvil/internet & $265(25.1)$ & $322(29.4)$ & $290(33.3)$ & $41(3.9)$ & $41(3.7)$ & $31(3.6)$ & $4(0.4)$ & 14(1.3) & $2(0.2)$ & $26.12 * * *$ \\
\hline 2. Llamadas ofensivas/insultantes móvil/internet & 204(19.3) & $242(22.1)$ & $220(25.3)$ & $27(2.6)$ & $28(2.6)$ & $31(3.6)$ & $4(0.4)$ & $10(0.9)$ & $2(0.2)$ & $18.06^{* *}$ \\
\hline 3. Agredir. grabar y colgar en Internet & $106(10.0)$ & 102(9.3) & $98(11.3)$ & $23(2.2)$ & $22(2.0)$ & $9(1.0)$ & $4(0.4)$ & $3(0.3)$ & $2(0.2)$ & $6.24 \mathrm{~ns}$ \\
\hline 4. Difundir fotos/vídeos privados & $159(15.0)$ & 206(18.8) & $263(30.3)$ & $17(1.6)$ & $10(0.9)$ & $19(2.2)$ & $7(0.7)$ & $9(0.8)$ & $3(0.3)$ & $79.43 * * *$ \\
\hline 5. Fotografiar en vestuarios. playa... y difundir & $116(11.0)$ & $133(12.2)$ & $138(15.9)$ & $15(1.4)$ & $12(1.1)$ & $10(1.2)$ & $8(0.8)$ & $4(0.4)$ & $2(0.2)$ & \\
\hline 6. Llamadas anónimas para asustar & 154(14.6) & $229(20.9)$ & $221(25.4)$ & $36(3.4)$ & $22(2.0)$ & $32(3.7)$ & $5(0.5)$ & $8(0.7)$ & $3(0.3)$ & $43.43^{* * *}$ \\
\hline 7. Chantajear/amenazar mediante & 113(10.7) & $130(11.9)$ & $121(13.9)$ & $16(1.5)$ & $16(1.5)$ & $10(1.2)$ & $2(0.2)$ & $8(0.7)$ & $1(0.1)$ & $11.55 \mathrm{~ns}$ \\
\hline llamadas/mensajes & & & & & & & & & & \\
\hline nente móvil/internet & $56(5.3)$ & $69(6.3)$ & $72(8.3)$ & $11(1.0)$ & $13(1.2)$ & $7(0.8)$ & $4(0.4)$ & $3(0.3)$ & $0(0.0)$ & $10.71 \mathrm{~ns}$ \\
\hline 9. Suplant & 182(17.2) & $276(25.2)$ & $254(29.2)$ & $32(3.0)$ & $42(3.8)$ & $34(3.9)$ & $4(0.4)$ & $9(0.8)$ & $3(0.3)$ & $47.39 * * *$ \\
\hline 10. Robar la contraseña & $229(21.7)$ & $347(31.7)$ & $348(40.0)$ & $44(4.2)$ & $73(6.7)$ & $77(8.9)$ & $11(1.0)$ & 18(1.6) & $4(0.5)$ & $116.26^{* * *}$ \\
\hline 11. Trucar fotos/vídeos y difundir & $120(11.4)$ & 151(13.8) & $176(20.3)$ & 14(1.3) & $15(1.4)$ & $20(2.3)$ & $7(0.7)$ & $10(0.9)$ & $3(0.3)$ & 38.1 \\
\hline 12. Aislar e & $94(8.9)$ & $122(11.2)$ & $109(12.5)$ & $13(1.2)$ & $22(2.0)$ & $15(1.7)$ & $2(0.2)$ & $6(0.5)$ & $0(0.0)$ & $02 *$ \\
\hline $\begin{array}{l}\text { 13. Chantajear para hacer algo y no difundir } \\
\text { intimidad }\end{array}$ & $55(5.2)$ & $89(8.1)$ & $71(8.2)$ & $9(0.9)$ & $14(1.3)$ & $7(0.8)$ & $3(0.3)$ & $3(0.3)$ & $0(0.0)$ & $12.95^{*}$ \\
\hline 14. Amenazar de muerte & $47(4.4)$ & $46(4.2)$ & $64(7.4)$ & $9(0.9)$ & $12(1.1)$ & $3(0.3)$ & $3(0.3)$ & $7(0.6)$ & $3(0.3)$ & $16.76^{* *}$ \\
\hline 15. Difamar y crear rumores para desprestigiar & 205(19.4) & $243(22.2)$ & $255(29.3)$ & $28(2.6)$ & $37(3.4)$ & $42(4.8)$ & $8(0.8)$ & 12(1.1) & $4(0.5)$ & $39.56 * * *$ \\
\hline
\end{tabular}

Notas: $\chi^{2}=$ Chi cuadrado, ${ }^{*} p<.05, * * p<.01$, *** $p<.001$, ns $=$ no significativo. Los resultados en "Nunca" son la diferencia de los expuestos en la tabla. La $\chi^{2}$ se calcula con las 4 formas de respuestas.

$\mathrm{Al}$ analizar el número de participantes que informa haber sufrido conductas de ciberbullying una o más veces en cada grupo de edad (ver Tabla 2), no se hallan diferencias significativas en ninguna conducta. El porcentaje de víctimas en los distintos niveles de edad es similar, manteniéndose estable de 12 a 18 años en las 15 conductas evaluadas. El porcentaje de víctimas en los 3 grupos de edad es similar en las 15 conductas.

$\mathrm{Al}$ analizar el número de participantes que indica haber realizado conductas de ciberbullying en cada grupo de edad (ver Tabla 3), en 5 de las 15 conductas evaluadas se observa un incremento a los 14 años, que se mantiene o aumenta de 16 a 18 años. Estas 5 conductas fueron: Difundir fotos privadas o comprometidas, o vídeos de alguien a través del móvil, Internet... $(12-13=1.3 \% ; 14-15=2.5 \% ; 16-18=$ $2.6 \%$ ); Chantejear o amenazar por medio de llamadas o mensajes $(12-13=1.3 \% ; 14-15=2.2 \% ; 16-18=3.1 \%)$; Acosar sexualmente a través del móvil o Internet $(12-13=0.7 \% ; 14$ $15=1.5 \% ; 16-18=1.1 \%)$; Robar la contraseña de algún chico/a, para impedir que puedan acceder a su blog o a su correo electrónico $(12-13=3.1 \% ; 14-15=5.4 \% ; 16-18=$ $5.3 \%$ ); Amenazar de muerte a algún chico/a o a su familia a través del móvil, las redes sociales... $(12-13=0.6 \% ; 14-15=$ $1.6 \% ; 16-18=1.5 \%)$. En 10 de las 15 conductas el porcentaje de agresores en los distintos rangos de edad es similar, y en 5 conductas hay más agresores a medida que aumenta la edad.

$\mathrm{Al}$ analizar el número de participantes que revela haber observado conductas de ciberbullying en cada grupo de edad 
(ver Tabla 4), se ponen de relieve diferencias significativas en función de la edad en 12 de las 15 conductas evaluadas, evidenciándose un aumento significativo con la edad en: Enviar mensajes ofensivos e insultantes a través del móvil o Internet $(12-13=29.4 \% ; 14-15=34.4 \% ; 16-18=37.1 \%)$; Robar la contraseña de algún chico/a, para impedir que pueda acceder a su blog o a su correo electrónico $(12-13=26.9 \%$; $14-15=$ $40 \% ; 16-18=49.4 \%)$; Difamar, diciendo por Internet cosas de otra persona que son mentira para desprestigiarla, difundiendo rumores para hacerle daño $(12-13=22.8 \%$; $14-15=$ $26.7 \%$; $16-18=34.6 \%$; Hacer llamadas ofensivas e insultantes a través del móvil o Internet $(12-13=22.3 \% ; 14-15=$ $25.6 \% ; 16-18=29.1 \%$; Firmar en el blog de algún chico/a haciendo comentarios difamatorios, mentiras o contando sus secretos $(12-13=20.6 \% ; 14-15=29.8 \% ; 16-18=33.4 \%)$; Hacer llamadas anónimas con el fin de asustar y provocar miedo a otro $(12-13=18.5 \% ; 14-15=23.6 \% ; 16-18=29.4 \%)$; Difundir fotos privadas o comprometidas o vídeos de alguien a través del móvil o Internet $(12-13=17.3 \%$; 14-15= $20.5 \%$; 16-18 = 32.8\%); Trucar fotos o vídeos de algún chico/a para difundirlas (redes sociales, YouTube...) y humillarle o reírse de él o ella $(12-13=13.4 \% ; 14-15=16.1 \%$; $16-$ $18=22.9 \%)$; Hacer fotos robadas en sitios como vestuarios, playa, servicios... y colgarlas en Internet o difundirlas por el móvil $(12-13=13.2 \% ; 14-15=13.7 \% ; 16-18=17.3 \%)$; Acosar para intentar aislar a algún chico/a de sus contactos en las redes sociales $(12-13=10.3 \% ; 14-15=13.7 \% ; 16-18=$ $14.2 \%$ ); Chantajear, obligando a algún chico/a a realizar cosas que no quería, a cambio de no divulgar sus cosas íntimas en Internet $(12-13=6.4 \% ; 14-15=9.7 \% ; 16-18=9 \%)$; Amenazar de muerte a algún chico/a o a su familia a través del móvil, las redes sociales $(12-13=5.6 \% ; 14-15=5.9 \% ; 16-18=$ $8 \%$ ). Por lo tanto, el porcentaje de participantes que observa las 12 conductas de ciberbullying aumenta con la edad.

Media de conductas de ciberbullying: nivel de victimización, agresión, observación y victimizaciónagresiva de 12 a 18 años

Los resultados del MANOVA evidencian diferencias significativas en función de la edad, Lambda de Wilks, $\Lambda=.985$, $F(6,6000)=7.52, p<.001$ (tamaño del efecto muy bajo, $\eta^{2}=$ $.007, r=.08)$. Los resultados de los análisis descriptivos y univariantes de los 4 indicadores de ciberbullying se presentan en la Tabla 5.

Tabla 5. Medias, desviaciones típicas, análisis de varianza, tamaño del efecto (Eta cuadrado) y post hoc (Bonferroni) en indicadores de ciberbullying en distintas edades.

\begin{tabular}{|c|c|c|c|c|c|c|}
\hline \multirow[t]{2}{*}{ Indicadores de Ciberbullying } & 12-13 años & 14-15 años & 16-18 años & \multirow{2}{*}{$F(2,3023)$} & \multirow[t]{2}{*}{$\eta^{2}$} & \multirow[t]{2}{*}{ Post hoc } \\
\hline & $M(D T)$ & $M(D T)$ & $M(D T)$ & & & \\
\hline Nivel de victimización & $0.84(2.21)$ & $0.81(2.06)$ & $0.87(2.04)$ & $0.18 \mathrm{~ns}$ & .000 & ns \\
\hline Nivel de agresión & $0.34(1.52)$ & $0.59(2.83)$ & $0.49(1.84)$ & $3.78^{*}$ & .003 & $1<2$ \\
\hline Nivel de observación & $2.83(4.40)$ & $3.51(4.72)$ & $3.97(4.08)$ & $16.07 * * *$ & .011 & $1<2<3$ \\
\hline Nivel de victimización agresiva & $1.18(3.26)$ & $1.41(4.13)$ & $1.37(3.46)$ & $1.19 \mathrm{~ns}$ & .001 & ns \\
\hline
\end{tabular}

Notas: $* p<.05, * * * p<.001, \mathrm{~ns}=$ no significativo.

Los resultados (ver Tabla 5) confirman diferencias significativas en el nivel de perpetración (media de conductas realizadas), observándose un aumento significativo de las conductas que los agresores realizan desde los 12-13 años a los 14-15 años. En el nivel de observación, también se confirma un aumento significativo en la media de conductas observadas a medida que aumenta la edad $(12-13<14-15<16-18)$. La media de conductas de victimización (media de conductas sufridas por las víctimas) y de victimización-agresiva (media de conductas sufridas/realizadas por víctimas-agresivas) fueron similares de 12 a 18 años. Agresores y observadores informan de un aumento del número de conductas de ciberbullying que realizan y observan, a medida que aumenta la edad.

\section{Discusión}

En primer lugar, los resultados confirman que tanto el porcentaje de víctimas en los 3 grupos de edad como la media de conductas de ciberbullying sufridas por las víctimas se mantiene estable entre 12 y 18 años. Estos resultados confirman la primera hipótesis y ratifican los obtenidos por la mayoría de los estudios que no han hallado diferencias en victimización durante la adolescencia (Bauman, 2010; Gofin y Avitzour, 2012; Lauren y Ratliffe, 2011; Ortega et al., 2008; Walrave y Heirman, 2011), y la juventud (Hemphill et al., 2012). Sin embargo, contradicen aquellos que han encontrado una disminución del porcentaje de víctimas (Dehue et al., 2008; Mishna et al., 2012), un menor nivel de victimización (Moore et al., 2012), o un aumento de las víctimas con la edad (Hinduja y Patchin, 2008; Kowalski y Limber, 2007; Monks et al., 2012). Las discrepancias entre los estudios se deben en gran medida a las diferentes edades de las muestras, los diferentes instrumentos utilizados o al intervalo de tiempo considerado en la evaluación.

En segundo lugar, los resultados muestran que el porcentaje de agresores se mantiene estable entre 12 y 18 años en 10 de las 15 conductas evaluadas, sin embargo, en 5 conductas el porcentaje de agresores aumenta con la edad. Además, la media de conductas de perpetración informadas por los agresores aumenta significativamente con la edad. Estos resultados refutan la hipótesis 2 , ya que se había hipotetizado que no habría cambios. Los resultados confirman los obtenidos en los estudios que han encontrado un aumento de agresores (Hinduja y Patchin, 2008; Mishna et al., 2012; Smith et al., 2008), y del nivel de agresión (Bauman, 2010; 
Lauren y Ratliffe, 2011; Ybarra y Mitchell, 2007) con la edad. Las discrepancias con los estudios que no han hallado diferencias en función de la edad o han hallado una disminución de los agresores pueden explicarse por las distintas edades de los participantes. En general cuando la muestra contiene estudiantes hasta los 18 años se confirma un aumento con la edad de algunas conductas de ciberbullying y de la cantidad de conductas que se perpetran.

En tercer lugar, los observadores informan de incrementos significativos con la edad en 12 conductas. En la mayoría de estas conductas se da un aumento significativo en cada grupo de edad, es decir, el porcentaje de participantes que observa conductas de ciberbullying aumenta con la edad. Además, la media de conductas de ciberbullying que observan (nivel de observación) también aumenta significativamente. Los resultados no confirman la tercera hipótesis (ya que no se esperaban cambios) y contradicen los hallazgos de Álvarez-García et al. (2011) que encontraron una disminución con la edad, aunque esto puede ser explicado por el menor rango de edad (12-16 años) de los participantes de su estudio.

La investigación aporta información precisa sobre los cambios que se dan en la violencia a través de las TIC, en el ciberbullying en estas edades, evidenciando o bien una situación similar en el trascurso de las edades según las víctimas (ausencia de cambios), o bien un incremento de diversas conductas confirmadas por los agresores, y los observadores/testigos de ciberbullying. Además, el estudio permite comparar la información desde todos los roles implicados (víctimas-agresores-observadores), lo que facilita la triangulación de los datos, aumentando la fiabilidad de los mismos. Los datos evidencian similar porcentaje de víctimas, un aumento de agresores en 5 conductas y de la media de conductas agresivas que los agresores realizan, y un incremento del número de observadores de 12 conductas de ciberbullying, cuando lo que deberíamos esperar es una disminución relacionada con la maduración personal y social en el último tramo de edad. Sin embargo, esta disminución no se produce, el problema se mantiene en el tiempo y/o aumenta en algunas conductas, lo que enfatiza la necesidad de prevención e intervención.

Los resultados obtenidos al analizar el cambio con la edad son relevantes porque evidencian que las conductas violentas a través de las TIC, las conductas de ciberbullying, se mantienen estables en el tiempo, lo que pone de relieve la necesidad de prevención/intervención durante toda la escolarización. Los resultados sugieren que si no se interviene de forma preventiva es esperable que el ciberbullying no solo no disminuya, sino que aumente entre los 12 y los 18 años. Los hallazgos ponen de relieve la necesidad de implementar intervenciones con la finalidad de prevenir y eliminar el acoso entre iguales. La elevada participación en situaciones de ciberbullying, así como el incremento progresivo de este fenómeno en todos los países del mundo, permite enfatizar la urgente necesidad de prevención e intervención. La intervención para prevenir la violencia entre iguales en todas sus modalidades debe incluir tanto al contexto escolar como al familiar.

La escuela es un contexto de gran influencia en el desarrollo humano, un ámbito desde el que prevenir y reducir la conducta violenta. En todos los centros escolares debe haber un protocolo de actuación para los casos de acoso, así como un plan de prevención de la violencia. Una propuesta de intervención en contextos educativos debe contener actuaciones genéricas dirigidas a mejorar la convivencia, actuaciones específicas cuando se detectan situaciones de ciberbullying incipientes, para evitar su consolidación (a través de la aplicación de un programa específico con intervenciones individuales y en el grupo de alumnos...), y actuaciones cuando se trata de situaciones consolidadas, para minimizar el impacto sobre los implicados, aportando apoyo terapéutico y protección a las víctimas, así como control a los agresores.

Puesto que la mejor forma de prevenir la violencia es fomentar la convivencia, en todos los centros educativos, desde educación infantil hasta el final de la educación formal, se deberían realizar programas para promover el desarrollo socioemocional, programas que estimulen competencias sociales y emocionales en los niños, niñas y adolescentes que afectan positivamente a la convivencia y la prevención de la violencia. Programas de intervención que fomenten: (1) La capacidad para cooperar; (2) La capacidad para resolver conflictos de forma constructiva; (3) La autoestima positiva, saludable, y ajustada a la realidad; (4) La empatía o capacidad para hacerse cargo de los estados emocionales de otras personas; y (5) El control de las emociones. No obstante, y aunque en los últimos años la sensibilidad hacia el tema de la convivencia ha aumentado considerablemente, la aplicación sistemática de estos programas en los centros educativos sigue siendo una asignatura pendiente.

En las últimas décadas se han diseñado y evaluado diversos programas antibullying, sin embargo, y a pesar de la gran relevancia social que está adquiriendo el ciberbulling son muy pocos los programas que se han focalizado en la prevención y/o reducción del acoso tecnológico. La revisión realizada únicamente ha permitido identificar tres programas evaluados experimentalmente, basados en la evidencia: (1) El Brief Internet Ciberbullying Prevention Program (Doane, 2011), implementado con estudiantes universitarios en Canadá, que potenció una disminución de las conductas de perpetración del ciberacoso y de las actitudes positivas hacia ese tipo de conductas, aumentando el conocimiento del ciberbullying; (2) El Programa ConRed, "Conocer, construir, convivir en Internet y las redes sociales" (del Rey, Casas y Ortega, 2012), implementado con adolescentes españoles, que estimuló una disminución de las conductas de ciberbullying, especialmente la cibervictimización, el uso abusivo de internet y la percepción de falso control de la información en las redes sociales; y (3) Cyberprogram 2.0 (Garaigordobil y Martínez-Valderrey, 2014), implementado con adolescentes de 13 a 15 años, que disminuyó las conductas de bullying/ciberbullying, diferentes tipos de violencia escolar (violencia física y verbal por parte del alumnado, conductas de exclusión social...), la agresivi- 
dad impulsiva-premeditada, y aumentó las conductas sociales positivas, la autoestima, la empatía, y del uso de estrategias de resolución de conflictos cooperativas.

Las estrategias de prevención del ciberbullying también deben incluir a la familia, ya que la supervisión parental es fundamental para erradicar este tipo de conductas. Los padres deben recibir información de los resultados de las evaluaciones que se llevan a cabo en el centro, y deben estar implicados cuando se producen situaciones de acoso presencial y tecnológico. Además, la familia es una fuente muy relevante de influencia en los valores morales y en la conducta social de los hijos e hijas. Entre los factores del contexto familiar que fomentan la convivencia y la prevención de la violencia se pueden destacar: (1) Unas relaciones entre padres e hijos basadas en el afecto positivo, en el amor, que fomenten el establecimiento de vínculos de apego seguros, ya que éstos aportan sentimientos de seguridad básica que favorecen el autocontrol de la conducta agresiva; (2) Padres que sean agentes trasmisores de valores socio-morales positivos, padres modelos de empatía y de conducta prosocial; (3) Padres que eduquen en la tolerancia a la frustración, en el valor del esfuerzo y la perseverancia, que sean capaces de decir "no", que planteen normas educativas claras y razonadas, supervisando su cumplimiento, que pongan límites que demoren la

\section{Referencias}

Álvarez-García, D., Nuñez, J. C., Álvarez, L., Dobarro, A., Rodríguez, C. y González-Castro, P. (2011). Violencia a través de las tecnologías de la información y la comunicación en estudiantes de secundaria [Violence through information and communication technologies in secondary students]. Anales de Psicología, 27(1), 221-231.

Bauman, S. (2010). Cyberbullying in a rural intermédiate school: an exploratory study. Journal of Early Adolescence, 30(6), 803-833. doi:10.1177/0272431609350927

Calvete, E., Orue, I., Estévez, A., Villardón, L. y Padilla, P. (2010). Cyberbullying in adolescents: Modalities and aggressors' profile. Computers in Human Behavior, 26, 1128-1135. doi:10.1016\%2Fj.chb.2010.03.017

Cassidy, W., Jackson, M. y Brown, K. (2009). Sticks and stones can break my bones, but how can pixels hurt me? School Psychology International, 30(4), 383-402. doi:10.1177/0143034309106948

Dehue, F., Bolman, C. y Völlink, T. (2008). Cyberbullying: Youngsters’ Experiences and parental perception. Cyberpsychology and Behavior, 11(2), 217-223. doi:10.1089/cpb.2007.0008

del Rey, R., Casas, J. A. y Ortega, R. (2012). El programa ConRed, una práctica basada en la evidencia [The ConRed program, an evidence-based practice]. Revista Cientifica de Educomunicación, 20(39), 129-138. doi: 10.3916/C39-2012-03-03

Doane, A. N. (2011). Testing of a brief Internet cyberbullying prevention program in college students. Tesis doctoral: Old Dominion University, Facultad de Filosofía y Psicología.

Garaigordobil, M. (2011). Prevalencia y consecuencias del cyberbullying [Prevalence and consequences of cyberbullying: A review]. International Journal of Psychology and Psychological Therapy, 11(2), 233-254.

Garaigordobil, M. (2013). Cyberbullying: Screening de acoso entre iguales [Cyberbullying: Screening of peer harassment]. Madrid: TEA.

Garaigordobil, M. y Martínez-Valderrey, V. (2014). Cyberprogram 2.0. Un programa de intervención para la prevenir y reducir el ciberbullying [Cyberprogram 2.0. An intervention program to prevent and reduce cyberbullying]. Madrid: Pirámide.

Garaigordobil, M. y Oñederra, J. A. (2010). La violencia entre iguales: Revisión teórica y estrategias de intervención [Peer harassment: Theoretical review and intervention strategies]. Madrid: Pirámide. gratificación inmediata y potencien la gratificación a largo plazo; y (4) Padres que controlen la exposición de sus hijos/as a modelos de conducta violentos tanto en la realidad como en los medios (TV, video-juegos, internet...).

Como limitación del estudio cabe destacar el uso de un autoinforme, por el sesgo de deseabilidad social que conlleva, aunque en este estudio al obtener información de forma triangular el sesgo en parte queda neutralizado. Por otro lado, es difícil hacer afirmaciones de evolución de la conducta violenta a través de la edad ciñéndonos a un diseño de investigación transversal, lo que permite sugerir el desarrollo de estudios longitudinales que permitan el seguimiento de los participantes en los distintos rangos de edad. Líneas futuras de investigación podrían analizar: (1) la prevalencia en edades más tempranas; (2) los efectos para víctimas, agresores y observadores; y (3) las conexiones con factores personales y familiares, que ayuden a identificar variables relevantes para diseñar estrategias de prevención.

Agradecimientos.- Financiado por el Ministerio de Economía y Competitividad (PSI2012-30956), el Dpto. Educación, Universidades e Investigación del Gobierno Vasco (IT638-13) y la Unidad de Formación e Investigación UPV/EHU (UFIPSIXXI-11/04).

Gofin, R. y Avitzour, M. (2012). Traditional versus internet bullying in junior high school students. Maternal and Child Health Journal, 16(8), 16251635. doi:10.1007/s10995-012-0989-8

Hemphill, S., Tollit, M. y Kotevski, A. (2012). Rates of bullying perpetration and victimisation: A longitudinal study of secondary school students in Victoria, Australia. Pastoral Care in Education, 30(2), 99-112. doi:10.1080/02643944.2012.679953

Hinduja, S. y Patchin, J. W. (2008). Cyberbullying: An exploratory analysis of factors related to offending and victimization. Deviant Behavior, 29, 129-156. doi:10.1080/01639620701457816

Juvoven, J. y Gross, E. (2008). Extending the school grounds?-Bullying experiences in cyberspace. Journal of School Health, 78(9), 496-505.

Katzer, C., Fetchenhauer, D. y Belschak, F. (2009). Cyberbullying: Who are the victims? Journal of Media Psychology, 21(1), 25-36. doi:10.1027/18641105.21.1.25

Kowalski, R. M. y Limber, S. P. (2007). Electronic bullying among middle school students. Journal of Adolescent Health 41, 22-30. doi:10.1016/j.jadohealth.2007.08.017

Lauren, M. y Ratliffe, K. (2011). Cyber Worlds: New Playgrounds for Bullying. Computers in the Schools, 28(2), 92-116. doi:10.1080/07380569.2011.575753

Mishna, F., Khoury-Kassabri, M., Gadalla, T. y Daciuk, J. (2012). Risk factors for involvement in cyber bullying: Victims, bullies and bullyvictims. Children and Youth Services Review, 34(1), 63-70. doi:10.1016/j.childyouth.2011.08.032

Monks, C. P., Robinson, S. y Worlidge, P. (2012). The emergence of cyberbullying: A survey of primary school pupils' perceptions and experiences. School Psychology International, 33(5), 477-491. doi:10.1177/0143034312445242

Moore, P. M., Huebner, E. S. y Hills, K. J. (2012). Electronic bullying and victimization and life satisfaction in middle school students. Social Indicators Research, 107(3), 429-447. doi:10.1007/s11205-011-9856-z

Ortega, R., Calmaestra, J. y Mora-Merchán, J. (2008). Cyberbullying. International Journal of Psychology and Psychological Therapy, 8(2), 183-192. 
Raskauskas, J. y Stoltz, A. D. (2007). Involvement in traditional and electronic bullying among adolescents. Developmental Psychology, 43(3), 564 575. doi:10.1037/0012-1649.43.3.564

Sakellariou, T., Carroll, A. y Houghton, S. (2012). Rates of cyber victimization and bullying among male Australian primary and high school students. School Psychology International, 33(5), 533-549. doi:10.1177/0143034311430374

Slonje, R. y Smith, P. (2008). Cyberbullying: Another main type of bullying? Personality and Social Sciences, 49, 147-154. doi:10.1111/j.14679450.2007.00611.x

Smith, P., Mahdavi, J., Carvalho, M., Fisher, S., Russell, S. y Tippett, N. (2008). Cyberbullying: its nature an impact in secondary school pupils.
Journal of Child Psychology and Psychiatry, 49(4), 376-385. doi:10.1111/j.1469-7610.2007.01846.x

Walrave, M. y Heirman, W. (2011). Cyberbullying: Predicting victimization and perpetration. Children \& Society, 25, 59-72. doi:10.1111/j.10990860.2009.00260.x

Williams, K. y Guerra, N. (2007). Prevalence and predictors of internet bullying. Journal of Adolescent Health, 41, 14-21. doi:10.1016/j.jadohealth.2007.08.018

Ybarra, M. y Mitchell, K. J. (2007). Prevalence and frequency of internet harassment instigation: Implications for adolescent health. Journal of $A d-$ olescent Health, 41, 189-195. doi:10.1016/j.jadohealth.2007.03.005

(Articulo recibido: 12-07-2013; revisado: 05-05-2014; aceptado: 05-09-2014)

Anexo 1. Conductas del Test Cyberbullying.

1. ¿Te han enviado mensajes ofensivos e insultantes a través del móvil o a través de Internet?

2. ¿Te han hecho llamadas ofensivas e insultantes a través del móvil o de Internet (skype...)?

3. ¿Te han agredido para grabarte y colgarlo en Internet?

4. ¿Han difundido fotos tuyas privadas, o comprometidas, o vídeos, a través de Internet o el móvil?

5. ¿Te han hecho fotos robadas en sitios como los vestuarios, playa, servicios y las han colgado en Internet o difundido por el móvil?

6. ¿Has recibido llamadas anónimas, con el fin de asustarte y provocarte miedo?

7. ¿Te han chantajeado o amenazado por medio de llamadas o mensajes?

8. ¿Te han acosado sexualmente a través del móvil o de Internet?

9. ¿Ha firmado alguien en tu blog, haciéndose pasar por ti, haciendo comentarios difamatorios, mentiras o contando tus secretos?

10. ¿Te han robado la contraseña, para impedir que puedas acceder a tu blog o a tu correo electrónico?

11. ¿Te han trucado tus fotos o vídeos para difundirlas a través de redes sociales o YouTube, para humillarte o reírse de ti?

12. ¿Te han acosado para intentar aislarte de tus contactos en las redes sociales?

13. ¿Te han chantajeado, obligándote a realizar cosas que no querías a cambio de no divulgar tus cosas íntimas en la red?

14. ¿Te han amenazado de muerte a ti o a tu familia a través del teléfono móvil, de las redes sociales o de otro tipo de tecnología?

15. ¿Te han difamado a través de Internet diciendo cosas de ti que son mentira para desprestigiarte? ¿Han difundido rumores sobre ti para hacerte daño?

Nota: Los ítems se aplican a la víctima (ha sufrido), al agresor (ha realizado) y al observador (ha visto). 